\title{
A GENERAL LEMMA FOR FIXED-POINT THEOREMS INVOLVING MORE THAN TWO MAPS \\ IN $D$-METRIC SPACES WITH APPLICATIONS
}

\section{B. C. DHAGE, SMRATI ARYA, and JEONG SHEOK UME}

Received 26 June 2002

\begin{abstract}
A general procedural lemma for fixed-point theorems for three and four maps in a $D$-metric space is proved, and it is further applied for proving the common fixed-point theorems of three and four maps in a $D$-metric space satisfying certain contractive conditions.
\end{abstract}

2000 Mathematics Subject Classification: 47H10.

1. Introduction. The concept of a $D$-metric space is introduced by the first author in [2]. A nonempty set $X$, together with a function $\rho: X \times X \times X \rightarrow$ $[0, \infty)$, is called a $D$-metric space with $D$-metric $\rho$ if $\rho$ satisfies the following properties:

(i) $\rho(x, y, z)=0 \Leftrightarrow x=y=z$ (coincidence),

(ii) $\rho(x, y, z)=\rho(p\{x, z, y\})$ (symmetry), where $p$ is a permutation function,

(iii) $\rho(x, y, z) \leq \rho(x, y, a)+\rho(x, a, z)+\rho(a, y, z)$ for all $x, y, z, a \in X$ (tetrahedral inequality).

A few details along with some specific examples of a $D$-metric space appear in [3].

A sequence $\left\{x_{n}\right\} \subset X$ is said to be convergent to a point $x \in X$ if

$$
\lim _{m, n \rightarrow \infty} \rho\left(x_{m}, x_{n}, x\right)=0 .
$$

Similarly, a sequence $\left\{x_{n}\right\} \subset X$ is called $D$-Cauchy if

$$
\lim _{m, n, p \rightarrow \infty} \rho\left(x_{m}, x_{n}, x_{p}\right)=0 .
$$

A complete $D$-metric space is one in which every $D$-Cauchy sequence converges to a point. Further, a subset $S$ of a $D$-metric space $X$ is called bounded if there exists a constant $k>0$ such that $\rho(x, y, z) \leq k$ for all $x, y, z \in S$, and the constant $k$ is called a $D$-bound of $S$. The infinimum of all such $D$-bounds $k$ is called the diameter of $S$, and it is denoted by $\delta(S)$. Finally, it is known that a 
mapping $f: X \rightarrow X$ is continuous if and only if, for any sequence $\left\{x_{n}\right\} \subset X$, $x_{n} \rightarrow x^{*}$ implies $f x_{n} \rightarrow f x^{*}$.

It has been shown in [5] that the $D$-metric $\rho$ is continuous on $X^{3}$ in the topology of $D$-metric convergence which is Hausdorff. For details of a $D$-metric space, the reader is referred to Dhage [5].

The general existence principles for the fixed-point theorems for a single and a pair of maps in $D$-metric space have been established in Dhage and Rhoades [6] and Dhage [1], respectively. However, the extension of these existence principles to three or four maps is not possible.

In this paper, we just formulate the general procedure for common fixedpoint theorems for more than two selfmaps of a $D$-metric space and discuss some of its applications.

We need the following auxiliary results in the sequence.

Proposition 1.1. Let $\left\{x_{n}\right\}$ be a sequence in a D-metric space $X$ satisfying

$$
\rho\left(x_{n}, x_{n+1}, z\right) \leq \lambda \rho\left(x_{n-1}, x_{n}, z\right)
$$

for all $n \in \mathbb{N}$ and $z \in\left\{x_{n}\right\}$, where $0 \leq \lambda<1$. Then,

$$
\rho\left(x_{n}, x_{n+1}, x_{m}\right) \leq \lambda^{n} k
$$

for all $m>n$, where $k=(2 /(1-\lambda)) \max \left\{\rho\left(x_{0}, x_{0}, x_{1}\right), \rho\left(x_{0}, x_{1}, x_{1}\right)\right\}$.

Proof. From (1.3),

$$
\rho\left(x_{n}, x_{n+1}, x_{m}\right) \leq \lambda \rho\left(x_{n-1}, x_{n}, x_{m}\right)
$$

for each $m>n \in \mathbb{N}$. By induction,

$$
\rho\left(x_{n}, x_{n+1}, x_{m}\right) \leq \lambda^{n} \rho\left(x_{0}, x_{1}, x_{m}\right) .
$$

Let $q=\max \left\{\rho\left(x_{0}, x_{0}, x_{1}\right), \rho\left(x_{0}, x_{1}, x_{1}\right)\right\}$. Using the tetrahedral inequality,

$$
\begin{aligned}
\rho\left(x_{0}, x_{1}, x_{m}\right) & \leq \rho\left(x_{0}, x_{1}, x_{m-1}\right)+\rho\left(x_{m-1}, x_{0}, x_{m}\right)+\rho\left(x_{m-1}, x_{m}, x_{1}\right) \\
& =\rho\left(x_{0}, x_{1}, x_{m-1}\right)+\rho\left(x_{m-1}, x_{m}, x_{0}\right)+\rho\left(x_{m-1}, x_{m}, x_{1}\right) \\
& \leq \rho\left(x_{0}, x_{1}, x_{m-1}\right)+\lambda^{m-1} \rho\left(x_{0}, x_{1}, x_{0}\right)+\lambda^{m-1} \rho\left(x_{0}, x_{1}, x_{1}\right) \\
& \leq \rho\left(x_{0}, x_{1}, x_{m-1}\right)+2 \lambda^{m-1} q \\
& \leq \rho\left(x_{0}, x_{1}, x_{m-2}\right)+2 \lambda^{m-1} q+2 \lambda^{m-2} q \\
& \vdots \\
& \leq \rho\left(x_{0}, x_{1}, x_{1}\right)+2\left(\lambda+\lambda^{2}+\cdots+\lambda^{m-1}\right) q \\
& \leq 2\left(1+\lambda+\lambda^{2}+\cdots+\lambda^{m-1}\right) q<\frac{2}{1-\lambda} q=k .
\end{aligned}
$$

Substituting (1.7) into (1.6) yields the desired inequality (1.4). 
Proposition 1.2. Every sequence $\left\{x_{n}\right\} \subset X$ satisfying (1.3) is bounded with a $D$-bound $k=(2 /(1-\lambda)) \max \left\{\rho\left(x_{0}, x_{0}, x_{1}\right), \rho\left(x_{0}, x_{1}, x_{1}\right)\right\}$.

Proof. Let $q=\max \left\{\rho\left(x_{0}, x_{0}, x_{1}\right), \rho\left(x_{0}, x_{1}, x_{1}\right)\right\}$. Then, for any integers $r \geq$ $s \geq n$, there exists positive integer $p$ and $t$ such that

$$
\begin{aligned}
\rho\left(x_{n}, x_{r}, x_{s}\right) & =\rho\left(x_{n}, x_{n+p}, x_{n+t}\right) \\
& \leq \rho\left(x_{n}, x_{n+1}, x_{n+t}\right)+\rho\left(x_{n}, x_{n+1}, x_{n+p}\right)+\rho\left(x_{n+1}, x_{n+p}, x_{n+t}\right) \\
& \leq 2 \lambda^{n} q+\rho\left(x_{n+1}, x_{n+p}, x_{n+q}\right) \\
& \leq 2 \lambda^{n} q+2 \lambda^{n+1} q+\rho\left(x_{n+2}, x_{n+p}, x_{n+t}\right) \\
& \vdots \\
& \leq 2\left(\sum_{j=1}^{n+p-2} \lambda^{j}\right) q+\rho\left(x_{n+p-1}, x_{n+p}, x_{n+r}\right) \\
& \leq 2\left(\sum_{j=1}^{n+p-1} \lambda^{j}\right) q<2\left(\sum_{j=1}^{\infty} \lambda^{j}\right) q<\frac{2}{1-\lambda} q=k .
\end{aligned}
$$

Then, $\left\{x_{n}\right\}$ is bounded and the proof is complete.

2. Main results. Before going to the main results of this paper, we state a lemma proved in Dhage [4].

LEMMA 2.1 (D-Cauchy principle). Let $\left\{y_{n}\right\}$ be a bounded sequence in $D$ metric space with $D$-bound $k$ satisfying

$$
\rho\left(y_{n}, y_{n+1}, y_{m}\right) \leq \lambda^{n} k
$$

for all $m>n \in \mathbb{N}$. Then, $\left\{y_{n}\right\}$ is $D$-Cauchy.

Let $A, B, S, T: X \rightarrow X$ be four maps such that

$$
A(X) \subseteq T(X), \quad B(X) \subseteq S(X) .
$$

Condition (2.2) ensures that it is possible to define a sequence $\left\{y_{n}\right\}$ in $X$ as follows. Let $x \in X$ be arbitrary. Then, in view of condition (2.2), there exists a sequence $\left\{x_{n}\right\}$ such that

$$
x_{0}=x, \quad A x_{2 n}=T x_{2 n+1}, \quad B x_{2 n+1}=S x_{2 n+2}, \quad n \geq 0 .
$$

Now, define $\left\{y_{n}\right\}$ in $X$ by

$$
y_{0}=S x_{0}, \quad y_{2 n+1}=T x_{2 n+1}, \quad y_{2 n+2}=S x_{2 n+2}, \quad n \geq 0 .
$$

A point $x \in X$ is called a coincidence point of two maps $A, B: X \rightarrow X$ if $A x=B x$, and in this case, the mappings $A$ and $B$ are called coincident on $X$. 
Similarly, a coincidence point of three or four maps on a $D$-metric space is defined.

LemmA 2.2. Let $A, B, S, T: X \rightarrow X$ satisfy (2.2), and let $\left\{y_{n}\right\} \subset X$ be defined by (2.4). Further, assume that $\left\{y_{n}\right\}$ is complete. Suppose that there exists a $\lambda \in[0,1)$ such that

$$
\rho\left(y_{n}, y_{n+1}, z\right) \leq \lambda \rho\left(y_{n-1}, y_{n}, z\right)
$$

for all $n \in \mathbb{N}$ and $z \in\left\{y_{n}\right\}$. Then, either

(a) A and $S$ have a coincidence point,

(b) $B$ and $T$ have a coincidence point,

(c) A, $S$, and $T$ have a coincidence point,

(d) $B, S$, and $T$ have a coincidence point, or

(e) $\left\{y_{n}\right\}$ converges to a point $u \in X$ and, for all $m>n \in \mathbb{N}$,

$$
\rho\left(y_{n}, y_{m}, u\right) \leq 2 \sum_{j=n}^{m} \lambda^{j} k, \quad \rho\left(y_{n}, u, u\right) \leq 2 \frac{\lambda^{n}}{1-\lambda} k,
$$

where $k=\delta\left(\left\{y_{n}\right\}\right)$.

Proof. Suppose that $y_{2 n}=y_{2 n+1}$ for some $n$. Then, $S x_{2 n}=T x_{2 n+1}=A x_{2 n}$ and (a) holds. Also, if $x_{2 n}=x_{2 n+1}$, then $T x_{2 n}=T x_{2 n+1}$ and so (c) holds. Similarly, if $y_{2 n+1}=y_{2 n+2}$ for some $n$, then it is shown analogously that (b) and (d) hold.

Suppose now that $y_{n} \neq y_{n+1}$ for each $n$. Then, from Proposition 1.1, it follows that

$$
\rho\left(y_{n}, y_{n+1}, y_{m}\right) \leq \lambda^{n} \rho\left(y_{0}, y_{1}, y_{m}\right) \leq \lambda^{n} k
$$

for all $m>n \in \mathbb{N}$. Now, an application of Lemma 2.1 yields that $\left\{y_{n}\right\}$ is $D$ Cauchy. Since $\left\{y_{n}\right\}$ is complete, there exists a point $u \in X$ such that $\lim _{n} y_{n}=u$.

Now, for any positive integers $m$ and $n, m>n$, by repeated application of the tetrahedral inequality,

$$
\begin{aligned}
\rho\left(y_{n}, y_{m}, u\right) & \leq \rho\left(y_{n}, y_{n+1}, y_{m}\right)+\rho\left(y_{n}, y_{n+1}, u\right)+\rho\left(y_{n+1}, y_{m}, u\right) \\
& \leq \lambda^{n} \rho\left(y_{0}, y_{1}, y_{m}\right)+\lambda^{n} \rho\left(y_{0}, y_{1}, u\right)+\rho\left(y_{n+1}, y_{m}, u\right) \\
& \leq 2 \lambda^{n} k+\rho\left(y_{n+1}, y_{m}, u\right) \\
& \leq 2 \lambda^{n} k+2 \lambda^{n+1} k+\rho\left(y_{n+2}, y_{m}, u\right) \\
& \vdots \\
& \leq 2\left(\lambda^{n}+\lambda^{n+1}+\cdots+\lambda^{m}\right) k \\
& =2 \sum_{j=n}^{m} \lambda^{j} k .
\end{aligned}
$$


The above inequality further gives that

$$
\begin{aligned}
\rho\left(y_{n}, y_{m}, u\right) & \leq 2 \sum_{j=n}^{m} \lambda^{j} k \\
& =2 \lambda^{n}\left(1+\lambda+\cdots+\lambda^{m-n}\right) k \\
& =2 \lambda^{n}\left(\frac{1-\lambda^{m-n}}{1-\lambda}\right) k .
\end{aligned}
$$

Taking the limit as $m \rightarrow \infty$ in the above inequality,

$$
\rho\left(y_{n}, u, u\right) \leq 2 \frac{\lambda^{n}}{1-\lambda} k
$$

The proof of Lemma 2.2 is complete.

The three-maps version of Lemma 2.2 is obtained in two ways: one by setting $S=T$ and the other by setting $A=B$. In the situation when $S=T$, condition (2.2) reduces to

$$
A(X) \subseteq S(X), \quad B(X) \subseteq S(X) .
$$

Then, it is possible to choose a sequence $\left\{x_{n}\right\} \subset X$ such that

$$
x_{0}=x, \quad A x_{2 n}=S x_{2 n+1}, \quad B x_{2 n+1}=S x_{2 n+2}, \quad n \geq 0 .
$$

Now, define a sequence $\left\{y_{n}\right\}$ in $X$ as follows:

$$
y_{0}=S x_{0}, \quad y_{2 n}=S x_{2 n}, \quad y_{2 n+1}=S x_{2 n+1}, \quad n \in \mathbb{N} \text {. }
$$

LEMмA 2.3. Let $A, B, S: X \rightarrow X$ satisfy (2.11). Suppose that there exists an $x \in X$ such that the sequence $\left\{y_{n}\right\} \subset X$ defined by (2.13) is complete. Further, suppose that

$$
\rho\left(y_{n}, y_{n+1}, z\right) \leq \lambda \rho\left(y_{n-1}, y_{n}, z\right)
$$

for all $n \in \mathbb{N}$ and $z \in\left\{y_{n}\right\}$, where $0 \leq \lambda<1$. Then, either

(a) A and $S$ have a coincidence point,

(b) $B$ and $S$ have a coincidence point, or

(c) $\left\{y_{n}\right\}$ converges to a point $u \in X$ and, for all positive integers $m$ and $n$, $m>n$,

$$
\rho\left(y_{n}, y_{m}, u\right) \leq 2 \sum_{j=n}^{m} \lambda^{j} k, \quad \rho\left(y_{n}, u, u\right) \leq 2 \frac{\lambda^{n}}{1-\lambda} k,
$$

where $k=\delta\left(\left\{y_{n}\right\}\right)$.

It is known that the fixed-point theorems for more than two maps require some sort of commutativity condition on the mappings under consideration. 
Below, we will apply Lemma 2.2 for proving the common fixed-point theorem for four maps on a $D$-metric space under a suitable commutativity condition.

A sequence $\left\{x_{n}\right\} \subset X$ is called a sequence of coincidence for the maps $A, B$ : $X \rightarrow X$ if $\lim _{n} A x_{n}=\lim _{n} B x_{n}$. In this case, the mappings $A$ and $B$ are called limit coincident on $X$. Similarly, two maps $A, B: X \rightarrow X$ are called commuting or commutative if $(A B)(x)=(B A)(x)$ for all $x \in X$ and limit commuting if there exists a sequence $\left\{x_{n}\right\} \subset X$ such that

$$
\lim _{n}(A B)\left(x_{n}\right)=\lim _{n}(B A)\left(x_{n}\right)
$$

Finally, two maps $A, B: X \rightarrow X$ are called limit coincidentally commuting if their limit coincidence implies the limit commuting on $X$, that is, for any sequence $\left\{x_{n}\right\} \subset X$ if

$$
\lim _{n} A x_{n}=\lim _{n} B x_{n} \Longrightarrow \lim _{n}(A B)\left(x_{n}\right)=\lim _{n}(B A)\left(x_{n}\right)
$$

It is known that the limit coincidentally commuting mappings commute at their coincidence points. See, for details, Dhage [4].

Now, we are ready to give some applications of Lemma 2.2 for proving the existence of a common fixed point of four maps on a $D$-metric space $X$.

An orbit of four selfmaps $A, B, S$, and $T$ of a $D$-metric space $X$ at a point $x \in X$ is a set $O_{A, B}(S, T: x)$ in $X$ defined by

$$
\begin{aligned}
& O_{A, B}(S, T: x)= \\
& \quad\left\{y_{0}=S x_{0}, y_{2 n+1}=A x_{2 n}=T x_{2 n+1}, y_{2 n+2}=B x_{2 n+1}=S x_{2 n+1}: n \geq 0\right\} .
\end{aligned}
$$

Clearly, the orbit $O_{A, B}(S, T: x)$ is well defined if $A, B, S$, and $T$ satisfy condition (2.2). By $\overline{O_{A, B}(S, T: x)}$, we denote the closure of the orbit $O_{A, B}(S, T: x)$ in $X$.

THEOREM 2.4. Let $A, B, S, T: X \rightarrow X$ be four selfmaps of a $D$-metric space $X$ satisfying (2.2) and

$$
\rho(A x, B y, z) \leq \lambda \max \{\rho(S x, T y, z), \rho(S x, A x, z), \rho(T y, B y, z)\}
$$

for all $x, y, z \in X$, where $0 \leq \lambda<1$. Assume further that

(a) $\overline{O_{A, B}(S, T: x)}$ is complete for each $x \in X$,

(b) $\{A, S\}$ and $\{B, T\}$ are limit coincidentally commuting,

(c) any one $A, B, S$, or $T$ continuous.

Then, $A, B, S$, and $T$ have a unique common fixed point.

Proof. Let $x \in X$ be arbitrary, and define a sequence $\left\{y_{n}\right\} \subset X$ by (2.4), which is possible in view of condition (2.4). Now, taking $x=x_{2 n}$ and $y=x_{2 n+1}$ 
in (2.19),

$$
\begin{aligned}
\rho\left(y_{2 n+1},\right. & \left.y_{2 n+2}, z\right) \\
\leq & \lambda \max \left\{\rho\left(y_{2 n}, y_{2 n+1}, z\right), \rho\left(y_{2 n}, y_{2 n+1}, z\right), \rho\left(y_{2 n+1}, y_{2 n+2}, z\right)\right\} \\
& =\lambda \rho\left(y_{2 n}, y_{2 n+1}, z\right)
\end{aligned}
$$

for all $n \geq 0$ and $z \in\left\{y_{n}\right\}$. Similarly, taking $x=x_{2 n}$ and $y=x_{2 n-1}$ in (2.19),

$$
\begin{aligned}
\rho\left(y_{2 n}, y_{2 n+1}, z\right) & \leq \lambda \max \left\{\rho\left(y_{2 n-1}, y_{2 n}, z\right), \rho\left(y_{2 n}, y_{2 n+1}, z\right), \rho\left(y_{2 n-1}, y_{2 n}, z\right)\right\} \\
& =\lambda \rho\left(y_{2 n-1}, y_{2 n}, z\right)
\end{aligned}
$$

for all $n \in \mathbb{N}$ and $z \in\left\{y_{n}\right\}$. Hence, in general,

$$
\rho\left(y_{n}, y_{n+1}, y_{m}\right) \leq \lambda \rho\left(y_{n-1}, y_{n}, y_{m}\right)
$$

for all $m>n \in \mathbb{N}$ and $0 \leq \lambda<1$.

We prove the conclusion of our theorem in two cases.

CASE 1. If $y_{n}=y_{n+1}$, then $y_{n}=y_{n+k}$ for all $k \geq 0$. If $y_{n+1} \neq y_{n+2}$, then, replacing $n$ in (2.22) by $n+1$,

$$
0<\rho\left(y_{n+1}, y_{n+2}, y_{n+1}\right) \leq \lambda \rho\left(y_{n}, y_{n+1}, y_{n+1}\right)=0 \text {, }
$$

which is a contradiction and $y_{n+1}=y_{n+2}$, and, by induction, $y_{n}=y_{n+k}$ for all $k \geq 0$. Therefore, by Lemma 2.2, there are points $u$ and $v$ in $X$ such that $w_{1}=A u=S u$ and $w_{2}=B v=T v$.

We will show that $w_{1}=w_{2}$. By (2.19),

$$
\begin{aligned}
\rho\left(w_{1}, w_{2}, w_{1}\right) & =\rho\left(A u, B v, w_{1}\right) \\
& \leq \lambda \max \left\{\rho\left(S u, T v, w_{1}\right), \rho\left(S u, A u, w_{1}\right), \rho\left(T v, B v, w_{1}\right)\right\} \\
& =\lambda \max \left\{\rho\left(w_{1}, w_{2}, w_{1}\right), \rho\left(w_{1}, w_{1}, w_{1}\right), \rho\left(w_{2}, w_{2}, w_{1}\right)\right\} \\
& =\lambda \rho\left(w_{1}, w_{2}, w_{2}\right) .
\end{aligned}
$$

Again,

$$
\begin{aligned}
\rho\left(w_{1}, w_{2}, w_{2}\right) & =\rho\left(A u, B v, w_{2}\right) \\
& \leq \lambda \max \left\{\rho\left(S u, T v, w_{2}\right), \rho\left(S u, A u, w_{2}\right), \rho\left(T v, B v, w_{2}\right)\right\} \\
& =\lambda \max \left\{\rho\left(w_{1}, w_{2}, w_{2}\right), \rho\left(w_{1}, w_{1}, w_{2}\right), \rho\left(w_{2}, w_{2}, w_{2}\right)\right\} \\
& =\lambda \rho\left(w_{1}, w_{2}, w_{1}\right) .
\end{aligned}
$$

Substituting (2.25) into (2.24),

$$
\rho\left(w_{1}, w_{2}, w_{1}\right) \leq \lambda^{2} \rho\left(w_{1}, w_{2}, w_{1}\right)
$$


which is possible only when $w_{1}=w_{2}$ since $\lambda<1$. Hence, $A u=B v=S u=$ $T v=w$. Next, we show that $w$ is a coincidence of $A, B, S$, and $T$. Since $\{A, S\}$ and $\{B, T\}$ are limit coincidentally commuting, they commute at coincidence point. Therefore, $S w=S A u=A S u=A w$ and $T w=T B v=B T v=B w$. Now,

$$
\begin{aligned}
\rho(A w, B w, A w) & \leq \lambda \max \{\rho(S w, T w, A w), \rho(S w, A w, A w), \rho(T w, B w, A w)\} \\
& =\lambda \max \{\rho(A w, B w, A w), \rho(B w, B w, A w)\} \\
& =\lambda \rho(A w, B w, B w) .
\end{aligned}
$$

Similarly,

$$
\rho(A w, B w, B w) \leq \lambda \rho(A w, B w, A w)
$$

Substituting (2.28) into (2.27),

$$
\rho(A w, B w, A w) \leq \lambda^{2} \rho(A w, B w, A w),
$$

which is possible only when $A w=B w$. Hence, $A w=S w=T w=B w$ is a coincidence point of the four maps $A, B, S$, and $T$. Finally, we prove that $w$ is a common fixed point of $A, B, S$, and $T$. If $w \neq A w$, then, by (2.19),

$$
\begin{aligned}
\rho(A w, w, w) & =\rho(A w, B v, w) \\
& \leq \lambda \max \{\rho(S w, T v, w), \rho(S w, A w, w), \rho(T v, B v, w)\} \\
& =\lambda \rho(A w, w, A w)
\end{aligned}
$$

Similarly,

$$
\rho(A w, w, A w) \leq \lambda \rho(A w, w, w) .
$$

From (2.30) and (2.31),

$$
\rho(A w, w, w) \leq \lambda^{2} \rho(A w, w, w),
$$

which is a contradiction to $A w=w$ and hence, $w=A w=S w=T w=B w$.

CASE 2. Suppose that $y_{n} \neq y_{n+1}$ for each $n$. Then, by Lemma 2.2, there exists a point $w \in X$ such that $\lim _{n} y_{n}=w$. By definition of $\left\{y_{n}\right\}$,

$$
\begin{aligned}
\lim _{n} y_{2 n} & =\lim _{n} S x_{2 n}=\lim _{n} A x_{2 n}=\lim _{n} T x_{2 n+1}=w, \\
\lim _{n} y_{2 n+1} & =\lim _{n} T x_{2 n+1}=\lim _{n} B x_{2 n+1}=\lim _{n} y_{2 n+2}=w .
\end{aligned}
$$

Since $\{A, S\}$ and $\{B, T\}$ are limit coincidentally commuting, then

$$
\begin{aligned}
\lim _{n} A S x_{2 n} & =\lim _{n} S A x_{2 n}, \\
\lim _{n} B T x_{2 n+1} & =\lim _{n} T B x_{2 n+1} .
\end{aligned}
$$


Suppose first that $S$ is continuous on $X$. Then,

$$
\lim _{n} S S x_{2 n}=\lim _{n} S A x_{2 n}=\lim _{n} A S x_{2 n}=S w .
$$

First, we show that $w$ is a fixed point of $S$. If $w \neq S w$, then, by (2.19),

$$
\begin{aligned}
& \rho(S w, w, w) \\
& =\lim _{n} \rho\left(A S x_{2 n}, B x_{2 n+1}, w\right) \\
& \leq \lambda \lim _{n} \max \left\{\rho\left(S S x_{2 n}, T x_{2 n+1}, w\right), \rho\left(S B x_{2 n}, A S x_{2 n}, w\right), \rho\left(T x_{2 n+1}, B x_{2 n+1}, w\right)\right\} \\
& =\lambda \max \{\rho(S w, w, w), \rho(S w, S w, w)\} \\
& =\lambda \rho(S w, S w, w) .
\end{aligned}
$$

Similarly,

$$
\rho(S w, w, S w) \leq \lambda \rho(S w, w, w)
$$

Substituting (2.37) into (2.36),

$$
\rho(S w, w, w) \leq \lambda^{2} \rho(S w, w, w)
$$

which is a contradiction and hence, $S w=w$. Similarly,

$$
\begin{aligned}
\rho(A w, w, w) \\
\quad \leq \lim _{n} \rho\left(A w, B x_{2 n+1}, w\right) \\
\leq \lambda \lim _{n} \max \left\{\rho\left(S w, T x_{2 n+1}, w\right), \rho\left(T x_{2 n+1}, B x_{2 n+1}, w\right), \rho(S w, A w, w)\right\} \\
=\lambda \max \{0,0, \rho(A w, w, w)\} \\
=\lambda \rho(A w, w, w),
\end{aligned}
$$

which implies that $A w=w$, since $\lambda<1$. From the condition $A(X) \subseteq T(X)$, it follows that there is a point $p \in X$ such that $w=A w=T p$. We show that $B p=T p$. If not, then

$$
\begin{aligned}
\rho(T p, B p, w) & =\rho(A w, B p, w) \\
& \leq \lambda \max \{\rho(S w, T p, w), \rho(S w, A w, w), \rho(T p, B p, w)\} \\
& =\lambda \rho(T p, B p, w)
\end{aligned}
$$

which is a contradiction. Hence, $B p=T p$. Since $\{B, T\}$ are limit coincidentally commuting, we obtain $B w=B T p=T B p=B w$. Now,

$$
\begin{aligned}
\rho(A w, B w, w) & \leq \lambda \max \{\rho(S w, T w, w), \rho(S w, A w, w), \rho(T w, B w, w)\} \\
& =\lambda \rho(A w, B w, B w) .
\end{aligned}
$$


Similarly,

$$
\rho(A w, B w, B w) \leq \lambda \rho(A w, B w, w) .
$$

Substituting (2.42) into (2.41),

$$
\rho(A w, B w, w) \leq \lambda^{2} \rho(A w, B w, w)
$$

which is possible only when $A w=B w$. Thus, $w$ is a common fixed point of $A$, $B, S$, and $T$.

Similarly, if $T$ is continuous, then it is proved in an analogous way that $A$, $B, S$, and $T$ have a common fixed point.

Next, suppose that $A$ is continuous. Then, we have

$$
\lim _{n} A A x_{2 n}=\lim _{n} A S x_{2 n}=\lim _{n} S A x_{2 n}=A w .
$$

First, we show that $A w=w$. If $A w \neq w$, then

$$
\begin{aligned}
& \rho(A w, w, w) \\
& =\lim _{n} \rho\left(A A x_{2 n}, B x_{2 n+1}, w\right) \\
& \leq \lambda \lim _{n} \max \left\{\rho\left(S A x_{2 n}, T x_{2 n+1}, w\right), \rho\left(S A x_{2 n}, A A x_{2 n}, w\right), \rho\left(T x_{2 n+1}, B x_{2 n+1}, w\right)\right\} \\
& =\lambda \max \{\rho(A w, w, w), \rho(A w, A w, w)\} \\
& =\lambda \rho(A w, A w, w) .
\end{aligned}
$$

Similarly,

$$
\rho(A w, w, A w) \leq \lambda \rho(A w, w, w) .
$$

Substituting (2.46) into (2.45),

$$
\rho(A w, w, w) \leq \lambda^{2} \rho(A w, w, w),
$$

which is a contradiction. Hence, $A w=w$. Using condition (2.2), there exists a point $p \in X$ such that $T p=A w=w$. We show that $B p=T p$. Now,

$$
\begin{aligned}
\rho(A w, B p, w) \\
\quad=\lim _{n} \rho\left(A A x_{2 n}, B p, w\right) \\
\leq \lambda \lim _{n} \max \left\{\rho\left(S A x_{2 n}, T p, w\right), \rho\left(S A x_{2 n}, A A x_{2 n}, w\right), \rho(T p, B p, w)\right\} \\
=\lambda \rho(w, B p, w),
\end{aligned}
$$


which gives that $B p=T p$. Since $\{B, T\}$ are limit coincidentally commuting, they commute at coincidence point. Hence, $T w=T B p=B T p=B w$. Now,

$$
\rho\left(A x_{2 n}, B w, w\right) \leq \lambda \max \left\{\rho\left(S x_{2 n}, T w, w\right), \rho\left(S x_{2 n}, A x_{2 n}, w\right), \rho(T w, B w, w)\right\}
$$

Taking the limit as $n \rightarrow \infty$,

$$
\rho(w, B w, w) \leq \lambda \rho(w, B w, B w)
$$

Similarly,

$$
\rho(w, B w, B w) \leq \lambda \rho(w, B w, w)
$$

Substituting (2.51) into (2.50),

$$
\rho(w, B w, w) \leq \lambda^{2} \rho(w, B w, w)
$$

which implies that $w=B w=T w=A w$. Since $B(X) \subseteq S(X)$, there is a point $q \in X$ such that $w=B w=S q$. We show that $A q=S q$. Now,

$$
\begin{aligned}
\rho(A q, S q, w) & =\rho(A q, B w, w) \\
& \leq \lambda \max \{\rho(S q, T w, w), \rho(S q, A q, w), \rho(T w, B w, w)\} \\
& =\lambda \rho(S q, A q, w),
\end{aligned}
$$

which implies that $A q=S q$. Since $\{A, S\}$ are limit coincidentally commuting $S w=S A q=A S q=A w=w$. Thus, $A w=S w=T w=B w$, that is, $w$ is a common fixed point of $A, B, S$, and $T$. Similarly, if $B$ is continuous, it is proved that $A, B, S$, and $T$ have a common fixed point.

To prove the uniqueness, let $w^{*}(\neq w)$ be common fixed point of $A, B, S$, and $T$. Then,

$$
\begin{aligned}
\rho\left(w, w^{*}, w^{*}\right) & =\rho\left(A w, B w^{*}, w^{*}\right) \\
& \leq \lambda \max \left\{\rho\left(S w, T w^{*}, w^{*}\right), \rho\left(S w, A w, w^{*}\right), \rho\left(T w^{*}, B w^{*}, w^{*}\right)\right\} \\
& =\lambda \rho\left(w, w, w^{*}\right)
\end{aligned}
$$

Similarly,

$$
\rho\left(w, w, w^{*}\right) \leq \lambda \rho\left(w, w^{*}, w^{*}\right)
$$

Substituting (2.54) into (2.55),

$$
\rho\left(w, w, w^{*}\right) \leq \lambda^{2} \rho\left(w, w, w^{*}\right),
$$

which is a contradiction. Hence, $w=w^{*}$. This completes the proof. 
Letting $S=T$ in Theorem 2.4, we obtain the following corollary.

COROLLARY 2.5. Let $A, B$, and $S$ be three selfmappings of a $D$-metric space satisfying (2.11) and

$$
\rho(A x, B y, z) \leq \lambda \max \{\rho(S x, S y, z), \rho(S x, A x, z), \rho(S y, B y, z)\}
$$

for all $x, y, z \in X$, where $0 \leq \lambda<1$.

Further assume that

(a) $\overline{O_{A, B}(S x)}$ is complete for each $x \in X$,

(b) $\{A, S\}$ and $\{B, S\}$ are limit coincidentally commuting,

(c) any one of $A, B$, and $S$ is continuous.

Then $A, B$, and $S$ have a unique common fixed point.

ACKNOWLEDGMENTS. We would like to express our thanks to the referee for his helpful suggestions. This work was supported by grant no. 2001-110100-005-2 from the Basic Research Program of the Korea Science and Engineering Foundation.

\section{REFERENCES}

[1] B. C. Dhage, On two general existence principles for proving the common fixed point theorems in D-metric spaces, to appear in Indian J. Pure Appl. Math.

[2] _ A study of some fixed point theorems, Ph.D. thesis, Marathwada University, Aurangabad, India, 1984.

[3] _ Generalised metric spaces and mappings with fixed point, Bull. Calcutta Math. Soc. 84 (1992), no. 4, 329-336.

[4] - On common fixed points of pairs of coincidentally commuting mappings in D-metric spaces, Indian J. Pure Appl. Math. 30 (1999), no. 4, 395-406.

[5] _ Generalized metric spaces and topological structure. I, An. Ştiinț. Univ. Al. I. Cuza Iaşi Secț. I a Mat. 46 (2000), no. 1, 3-24.

[6] B. C. Dhage and B. E. Rhoades, Proving fixed point theorems in D-metric spaces via general existence principle, to appear in Indian J. Pure Appl. Math.

B. C. Dhage: Gurukul Colony, Ahmedpur-413 515, Latur District (Maharashtra), India E-mail address: bcd20012001@yahoo.co. in

Smrati Arya: Chhatrapati Shivaji Ward, Kothari Gali, Harda-461331, India

Jeong Sheok Ume: Department of Applied Mathematics, Changwon National University, Changwon 641-773, Korea

E-mail address: jsume@changwon.ac. kr 


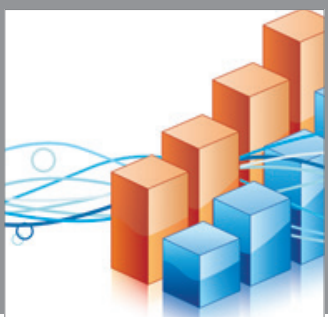

Advances in

Operations Research

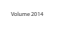

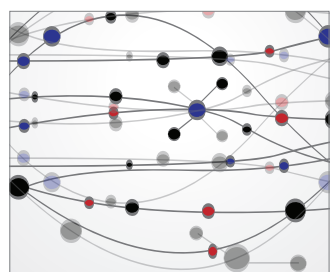

\section{The Scientific} World Journal
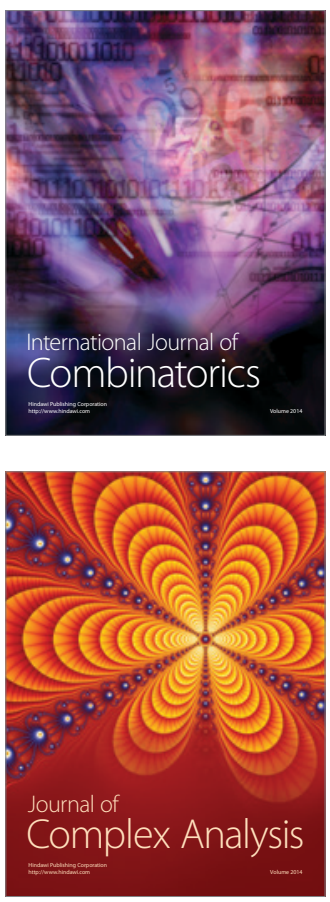

International Journal of

Mathematics and

Mathematical

Sciences
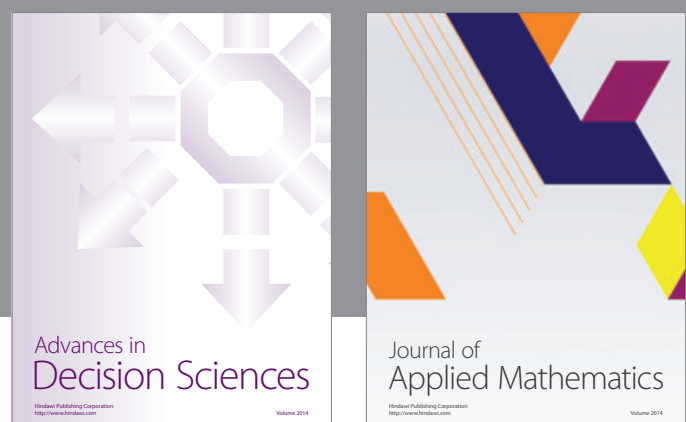

Journal of

Applied Mathematics
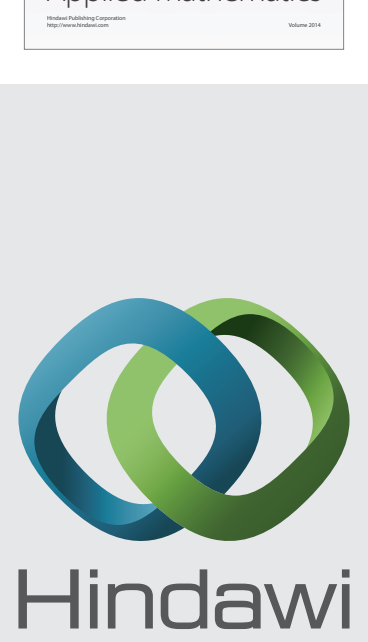

Submit your manuscripts at http://www.hindawi.com
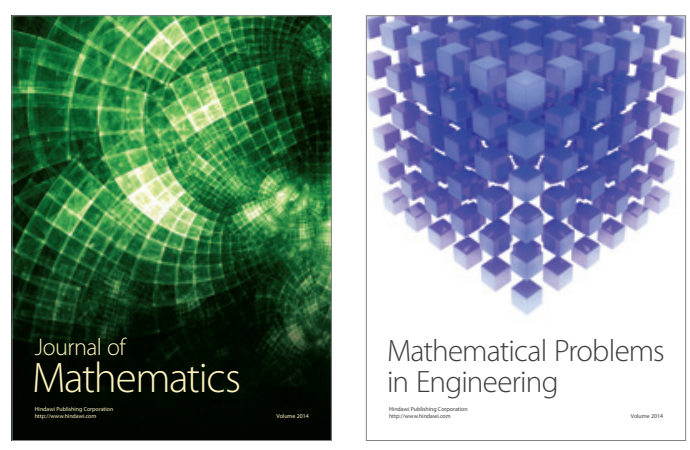

Mathematical Problems in Engineering
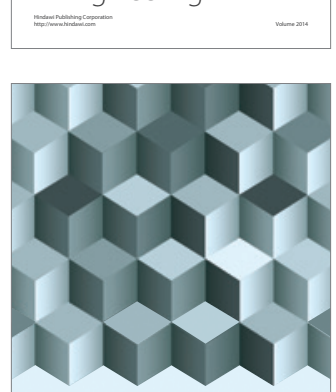

Journal of

Function Spaces
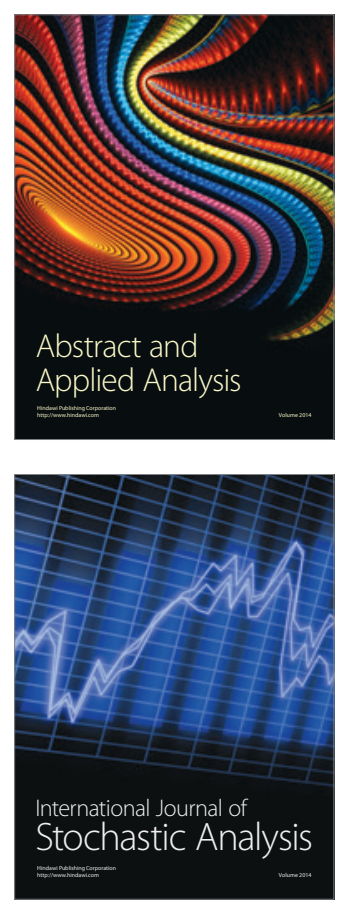

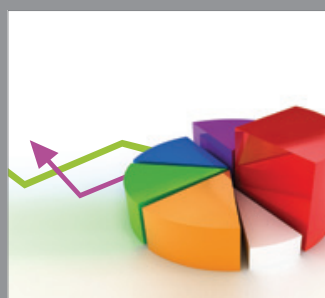

ournal of

Probability and Statistics

Promensencen
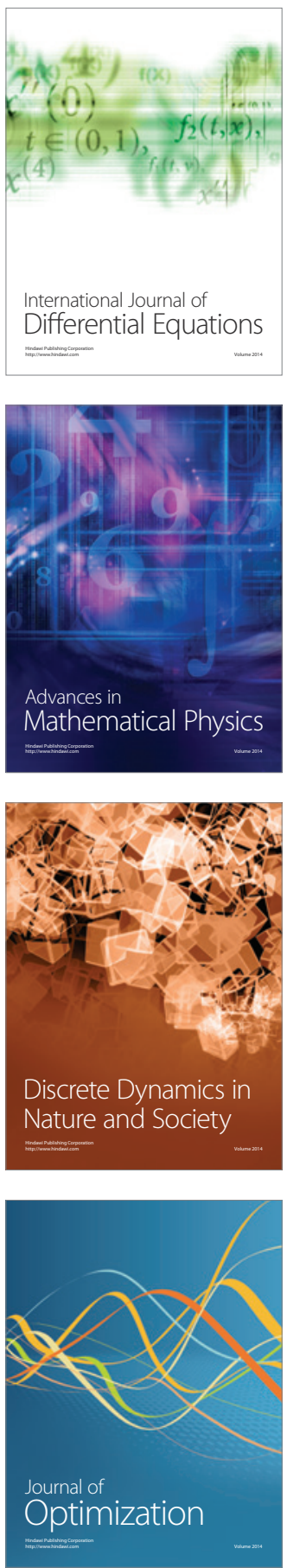